PROCEEDINGS OF THE

AMERICAN MATHEMATICAL SOCIETY

Volume 140, Number 2, February 2012, Pages 651-663

S 0002-9939(2011)10937-7

Article electronically published on June 21, 2011

\title{
COMPARISON PRINCIPLES FOR SELF-SIMILAR POTENTIAL FLOW
}

\author{
GUI-QIANG G. CHEN AND MIKHAIL FELDMAN \\ (Communicated by Walter Craig)
}

\begin{abstract}
We establish a strong comparison principle, as well as a weak comparison principle and a Hopf-type lemma, for elliptic solutions of the selfsimilar potential flow equation. A major difference from the steady case is that the coefficients of the equation depend on the potential function itself, as well as its gradient. We employ the divergence structure and other features of the equation to derive the results.
\end{abstract}

\section{INTRODUCTION}

We are concerned with unsteady potential flow in the spatial dimension $n \geq 2$. The Euler equations for potential flow consist of the conservation law of mass and Bernoulli's law for the density $\rho$ and velocity potential $\Phi$ :

$$
\begin{aligned}
& \partial_{t} \rho+\operatorname{div}_{\mathbf{x}}\left(\rho \nabla_{\mathbf{x}} \Phi\right)=0, \\
& \partial_{t} \Phi+\frac{1}{2}\left|\nabla_{\mathbf{x}} \Phi\right|^{2}+h(\rho)=K,
\end{aligned}
$$

where $(t, \mathbf{x}) \in \mathbb{R}_{+} \times \mathbb{R}^{n}, K$ is the Bernoulli constant, and $h(\rho)$ is defined from the prescribed pressure $p \in C([0, \infty)) \cap C^{3}((0, \infty))$,

$$
p^{\prime}(\rho)>0 \quad \text { for all } \rho>0,
$$

as follows:

$$
h^{\prime}(\rho)=\frac{p^{\prime}(\rho)}{\rho}=\frac{c^{2}(\rho)}{\rho}
$$

with $c(\rho)$ being the sound speed. See [3, 6, 7, 8, 11, 12, 13, 14] and the references cited therein.

For a polytropic gas,

$$
p(\rho)=\kappa \rho^{\gamma}, \quad c^{2}(\rho)=\kappa \gamma \rho^{\gamma-1}, \quad \gamma>1, \kappa>0 .
$$

Without loss of generality, we choose $\kappa=1 / \gamma$ so that

$$
h(\rho)=\frac{\rho^{\gamma-1}-1}{\gamma-1}, \quad c^{2}(\rho)=\rho^{\gamma-1}
$$

Received by the editors April 26, 2010 and, in revised form, December 4, 2010.

2010 Mathematics Subject Classification. Primary 35B51, 76N15, 76G25, 35Q35, 35J62, $35 \mathrm{~L} 65$.

Key words and phrases. Comparison principle, Hopf-type lemma, maximum principle, selfsimilar, potential flow, velocity potential, divergence structure.

(C)2011 American Mathematical Society 
which can be achieved by the following scaling:

$$
(t, \mathbf{x}, K) \rightarrow\left(\alpha^{2} t, \alpha \mathbf{x}, \alpha^{-2} K\right), \quad \alpha^{2}=\kappa \gamma .
$$

While our primary concern is with polytropic gases, in this paper we consider a more general class of pressure laws. The conditions on $p(\rho)$ will be stated below.

In many physically relevant problems, such as shock reflection-diffraction problems (cf. [1, 2, 3, 4, 5, 11, 14]) and problems involving supersonic flow onto solid wedges (cf. [8, 9]), self-similar solutions of potential flow of the form

$$
\rho(t, \mathbf{x})=\rho(\mathbf{y}), \quad \Phi(t, \mathbf{x})=t \phi(\mathbf{y}) \quad \text { for } \quad \mathbf{y}=\left(y_{1}, \ldots, y_{n}\right)=\frac{\mathbf{x}}{t}
$$

are important. Then the pseudo-potential function $\varphi(\mathbf{y})=\phi(\mathbf{y})-\frac{1}{2}|\mathbf{y}|^{2}$ satisfies the following Euler equations for self-similar solutions:

$$
\begin{aligned}
& \operatorname{div}(\rho D \varphi)+n \rho=0, \\
& \frac{1}{2}|D \varphi|^{2}+\varphi+h(\rho)=K,
\end{aligned}
$$

where the divergence div and gradient $D=\left(\partial_{y_{1}}, \ldots, \partial_{y_{n}}\right)$ are with respect to the self-similar variables $\mathbf{y}$. This implies that the pseudo-potential function $\varphi(\mathbf{y})$ is governed by the following potential flow equation of second order:

$$
\operatorname{div}\left(\rho\left(|D \varphi|^{2}, \varphi\right) D \varphi\right)+n \rho\left(|D \varphi|^{2}, \varphi\right)=0
$$

with

$$
\rho\left(|D \varphi|^{2}, \varphi\right)=\hat{\rho}\left(K-\left(\varphi+\frac{1}{2}|D \varphi|^{2}\right)\right)
$$

Here

$$
\hat{\rho}(s):=h^{-1}(s) \quad \text { for } s \geq h(0),
$$

where $h^{-1}(s)$ on $\{s \geq h(0)\}$ is the inverse function to $h(\rho)$, which is well-defined since $h^{\prime}(\rho)>0$ for $\rho>0$ by (1.3)-(1.4). It also follows that

$$
\hat{\rho}(s)>0, \quad \hat{\rho}^{\prime}(s)=\frac{1}{h^{\prime}(\hat{\rho}(s))}>0 \quad \text { for } s>h(0) .
$$

Consider solutions $\varphi$ of (1.7) satisfying $\rho\left(|D \varphi|^{2}, \varphi\right)>0$. Then, for the function $c^{2}(\rho)=p^{\prime}(\rho)$ in (1.4), define

$$
c^{2}\left(|D \varphi|^{2}, \varphi\right)=p^{\prime}\left(\rho\left(|D \varphi|^{2}, \varphi\right)\right)
$$

through (1.8). Then, from (1.4) and (1.9)-(1.10), we have

$$
c^{2}\left(|D \varphi|^{2}, \varphi\right)=\frac{\hat{\rho}}{\hat{\rho}^{\prime}}\left(K-\left(\varphi+\frac{1}{2}|D \varphi|^{2}\right)\right) .
$$

Now equation (1.7) can be written in the nondivergence form:

$$
\sum_{i, j=1}^{n}\left(c^{2}\left(|D \varphi|^{2}, \varphi\right) \delta_{i j}-\partial_{y_{i}} \varphi \partial_{y_{j}} \varphi\right) \partial_{y_{i} y_{j}} \varphi-|D \varphi|^{2}+n c^{2}\left(|D \varphi|^{2}, \varphi\right)=0,
$$

where $\delta_{i j}$ is the Kronecker delta with $\delta_{i i}=1$ and $\delta_{i j}=0$ for $i \neq j$.

Equation (1.7), equivalently (1.12) for smooth solutions, is a nonlinear equation of mixed elliptic-hyperbolic type. It is elliptic if and only if

$$
|D \varphi|<c\left(|D \varphi|^{2}, \varphi\right) \text {. }
$$


For the polytropic gas with $p=\rho^{\gamma} / \gamma, \gamma \geq 1$, (1.13) takes the form

$$
|D \varphi|<c_{*}(\varphi):=\sqrt{\frac{2}{\gamma+1}(\hat{K}-(\gamma-1) \varphi)},
$$

where $\hat{K}=1+(\gamma-1) K$.

A major difference from the steady case is that the coefficients of the self-similar potential flow equation written either in the divergence form (1.7) or in the nondivergence form (1.12) depend on the potential function $\varphi$ itself, as well as $D \varphi$. Thus, it is not clear whether comparison principles hold for elliptic solutions of (1.7). In this paper, we study comparison principles for (1.7), motivated by the applications to the shock reflection problem [3, 4, 5, and other problems in gas dynamics. An important feature in these problems is that ellipticity degenerates near a part of the boundary of the elliptic region, the sonic curve. Thus our results in this paper allow such an elliptic degeneracy.

We consider the pressure laws $p(\rho)$ satisfying condition (1.3) and

$$
F(\rho) \geq-2, \quad F^{\prime}(\rho) \leq 0 \quad \text { on }\{\rho>0\},
$$

where $F(\rho)=\frac{\rho p^{\prime \prime}(\rho)}{p^{\prime}(\rho)}$. The pressure law $p(\rho)=\rho^{\gamma} / \gamma$ for $\gamma \geq-1$ and $\gamma \neq 0$ satisfies (1.3) and (1.15), since $F(\rho)=\gamma-1$ in this case.

Define $\mathcal{N}$ as the nonlinear differential operator on the left-hand side of (1.7):

$$
\mathcal{N}(u):=\operatorname{div}\left(\rho\left(|D u|^{2}, u\right) D u\right)+n \rho\left(|D u|^{2}, u\right) .
$$

The main theorem of this paper is the following.

Theorem 1.1. Let $p \in C([0, \infty)) \cap C^{3}((0, \infty))$ satisfy (1.3) and (1.15) on $\{\rho>0\}$. Let $\Omega \subset \mathbb{R}^{n}$ be an open bounded set. Let $u, v \in C^{2}(\Omega) \cap C^{0,1}(\bar{\Omega})$ satisfy

$$
\mathcal{N}(u) \geq 0, \quad \mathcal{N}(v) \leq 0 \quad \text { in } \Omega .
$$

Furthermore, assume that

$$
\begin{aligned}
& \rho\left(|D u|^{2}, u\right)>0, \quad|D u|^{2}<c^{2}\left(|D u|^{2}, u\right), \\
& \rho\left(|D v|^{2}, v\right)>0, \quad|D v|^{2}<c^{2}\left(|D v|^{2}, v\right)
\end{aligned}
$$

in $\Omega$. Then, if $u \leq v$ on $\partial \Omega$, it follows that $u \leq v$ in $\Omega$; furthermore, either $u<v$ in $\Omega$ or $u \equiv v$ in $\Omega$.

We also establish a Hopf-type lemma for the nonlinear self-similar potential flow equation (Lemma 4.1), as well as comparison principles for the linearized self-similar potential flow equation (Theorem 5.1 below).

Theorem 1.1 is obtained by employing a comparison principle for nonuniformly elliptic equations in the divergence form (Proposition 2.1 below), which is an extension of the comparison principle in Gilbarg-Trudinger [10, Theorem 10.7(iii)] to a class of equations of more general structure both including (1.16) and allowing a certain elliptic degeneracy. See Remark 2.2 for further details. Moreover, since equation (1.7) is of mixed type, then, when connecting two solutions $u$ and $v$, we have to show that this is done in the elliptic region of operator (1.16). For that, we use a convexity property of the ellipticity condition (1.13), which is implied by condition (1.15). See the proof of Theorem 3.1. 
From Lemma 3.2(ii), it follows that if the basic condition (1.3) holds, then condition (1.15) is necessary for the convexity properties of the ellipticity condition (1.13) used in the proof.

We remark in passing that the following ellipticity principle was established in Elling-Liu [8]: The interior of a parabolic-elliptic region of a sufficiently smooth solution must be elliptic; in fact, the local Mach number must be bounded above away from 1 by a region-dependent function. In particular, there are no open parabolic regions. This ellipticity principle plays an important role in the analysis of supersonic flow onto a solid wedge in 9 .

The rest of this paper is organized as follows. We first establish a weak comparison principle for nonuniformly elliptic equations in the divergence form in Section 2 and then apply it to the self-similar potential flow equation in Section 3. In Section 4, we establish the Hopf-type lemma for two elliptic solutions of (1.7) and apply it to establish the strong comparison principle, Theorem 1.1. In Section 5, as a concluding remark, we also show the weak and strong comparison principles for the linearized potential flow equation in the elliptic region.

\section{A COMPARISON PRINCIPLE FOR NONUNIFORMLY ELLIPTIC EQUATIONS} OF DIVERGENCE FORM

Let $\Omega \subset \mathbb{R}^{n}$ be open. We consider the nonlinear equation

$$
Q u=0,
$$

where

$$
Q u:=\operatorname{div}(\boldsymbol{A}(D u, u, \mathbf{y}))+B(D u, u, \mathbf{y})
$$

with $\boldsymbol{A}=\left(A^{1}, \ldots, A^{n}\right)$. The functions $\boldsymbol{A}(\boldsymbol{q}, z, \mathbf{y})$ and $B(\boldsymbol{q}, z, \mathbf{y})$ are defined on a subset of $\mathbb{R}^{n} \times \mathbb{R} \times \Omega$.

Let $u \in C^{1}(\Omega)$. For $S \subset \Omega$, denote

$$
\mathcal{E}(u, S):=\{(\boldsymbol{q}, z, \mathbf{y}): \boldsymbol{q}=D u(\mathbf{y}), z=u(\mathbf{y}), \mathbf{y} \in S\} \subset \mathbb{R}^{n} \times \mathbb{R} \times \Omega .
$$

Definition 2.1. Let $u \in C^{1}(\Omega) \cap C^{0,1}(\bar{\Omega})$. We say $Q u \geq 0$ (resp. $\leq 0$ ) in the weak sense if the functions $\boldsymbol{A}(\boldsymbol{q}, z, \mathbf{y})$ and $B(\boldsymbol{q}, z, \mathbf{y})$ are bounded on $\mathcal{E}(u, \Omega)$ and continuous in a neighborhood of $\mathcal{E}(u, S)$ for any compact set $S \subset \Omega$, and

$$
\int_{\Omega}(\boldsymbol{A}(D u, u, \mathbf{y}) \cdot D \zeta-B(D u, u, \mathbf{y}) \zeta) d \mathbf{y} \leq 0 \quad(\text { resp. } \geq 0)
$$

for all nonnegative functions $\zeta \in C_{c}^{1}(\Omega)$.

Also, for $u, v \in C^{1}(\Omega) \cap C^{0,1}(\bar{\Omega})$, denote

$\mathcal{E}([u, v], \Omega):=\left\{(\boldsymbol{q}, z, \mathbf{y}): \mathbf{y} \in \Omega, \boldsymbol{q}=D u_{t}(\mathbf{y}), z=u_{t}(\mathbf{y}), t \in[0,1]\right\} \subset \mathbb{R}^{n} \times \mathbb{R} \times \Omega$, where $u_{t}(\mathbf{y})=t u(\mathbf{y})+(1-t) v(\mathbf{y})$.

Proposition 2.1. Let $\Omega \subset \mathbb{R}^{n}$ be an open bounded set. Let $u, v \in C^{1}(\Omega) \cap C^{0,1}(\bar{\Omega})$ satisfy

$$
Q u \geq 0, \quad Q v \leq 0 \quad \text { in } \Omega
$$

in the weak sense. Assume that the functions $\boldsymbol{A}(\boldsymbol{q}, z, \mathbf{y})$ and $B(\boldsymbol{q}, z, \mathbf{y})$ satisfy the following: 
(i) $\boldsymbol{A}(\boldsymbol{q}, z, \mathbf{y})$ and $B(\boldsymbol{q}, z, \mathbf{y})$ are bounded on $\mathcal{E}([u, v], \Omega)$, and $D_{\boldsymbol{q}, z}(A, B)$ exist and are continuous in a neighborhood of $\mathcal{E}([u, v], S)$ for any compact set $S \subset \Omega$.

(ii) There exists $\beta \in(0,1]$ such that the $(n+1) \times(n+1)$ matrix

$$
H=\left[H_{i j}\right]:=\left[\begin{array}{ll}
\partial_{q_{i}} A^{j}(\boldsymbol{q}, z, \mathbf{y}) & -\beta \partial_{q_{i}} B(\boldsymbol{q}, z, \mathbf{y}) \\
\partial_{z} A^{j}(\boldsymbol{q}, z, \mathbf{y}) & -\beta \partial_{z} B(\boldsymbol{q}, z, \mathbf{y})
\end{array}\right]
$$

is nonnegative; that is,

$$
\sum_{i, j=1}^{n+1} H_{i j}(\boldsymbol{q}, z, \mathbf{y}) \xi_{i} \xi_{j} \geq 0
$$

for any $(\boldsymbol{q}, z, \mathbf{y}) \in \mathcal{E}([u, v], \Omega)$ and any $\boldsymbol{\xi}=\left(\boldsymbol{\xi}^{\prime}, \xi_{n+1}\right) \in \mathbb{R}^{n+1}$.

(iii) Moreover, for each $(\boldsymbol{q}, z, \mathbf{y}) \in \mathcal{E}([u, v], \Omega)$,

$$
\sum_{i, j=1}^{n+1} H_{i j}(\boldsymbol{q}, z, \mathbf{y}) \xi_{i} \xi_{j}>0
$$

for all $\boldsymbol{\xi}=\left(\boldsymbol{\xi}^{\prime}, \xi_{n+1}\right) \in \mathbb{R}^{n+1}$ with $\boldsymbol{\xi}^{\prime} \neq 0$.

Then, if $u \leq v$ on $\partial \Omega$, it follows that $u \leq v$ in $\Omega$.

Proof. In Definition 2.1, by approximation, we obtain that (2.3) also holds for all nonnegative functions $\zeta \in H_{0}^{1}(\Omega)$.

From the assumptions, the function $w=u-v$ satisfies

$$
w^{+}:=\max (w, 0) \in H_{0}^{1}(\Omega) \cap C^{0,1}(\bar{\Omega}) \cap C^{1}\left(\left\{w^{+}>0\right\} \cap \Omega\right) .
$$

Let $\zeta=\left(w^{+}\right)^{\frac{1}{\beta}}$. Since $\frac{1}{\beta}>1$, then $\zeta \in H_{0}^{1}(\Omega)$. Using this function $\zeta$ in (2.3) for $u$ and $v$, we obtain

$$
\begin{aligned}
\int_{\Omega}\{ & (\boldsymbol{A}(D u, u, \mathbf{y})-\boldsymbol{A}(D v, v, \mathbf{y})) \cdot D\left(w^{+}\right)^{\frac{1}{\beta}} \\
& \left.-(B(D u, u, \mathbf{y})-B(D v, v, \mathbf{y}))\left(w^{+}\right)^{\frac{1}{\beta}}\right\} d \mathbf{y} \leq 0 .
\end{aligned}
$$

Denote by $G(\mathbf{y})$ the integrand on the left-hand side. Our assumptions imply that $G \in C(\Omega) \cap L^{\infty}(\Omega)$. Also, using assumption (ii), we find that, in $\Omega$,

$$
G=\frac{1}{\beta}\left(w^{+}\right)^{\frac{1}{\beta}-1} \sum_{i, j=1}^{n}\left(a_{i j} \partial_{y_{i}} w^{+} \partial_{y_{j}} w^{+}+b_{i} w^{+} \partial_{y_{i}} w^{+}-\beta c_{i} w^{+} \partial_{y_{i}} w^{+}-\beta d\left(w^{+}\right)^{2}\right),
$$

with $a_{i j}, b_{j}, c_{i}, d \in C(\Omega)$ defined by

$$
\left(a_{i j}, b_{j}, c_{i}, d\right)(\mathbf{y})=\int_{0}^{1}\left(\partial_{q_{i}} A^{j}, \partial_{z} A^{j}, \partial_{q_{i}} B, \partial_{z} B\right)\left(D u_{t}(\mathbf{y}), u_{t}(\mathbf{y}), \mathbf{y}\right) d t
$$

for $\mathbf{y} \in \Omega$, where $u_{t}(\mathbf{y})=t u(\mathbf{y})+(1-t) v(\mathbf{y})$. Then (2.6) implies that $G \geq 0$ in $\Omega$. Thus, from (2.8), we obtain $G \equiv 0$ in $\Omega$. Therefore, on $\left\{w^{+}>0\right\} \cap \Omega$,

$$
\sum_{i, j=1}^{n}\left(a_{i j} \partial_{y_{i}} w^{+} \partial_{y_{j}} w^{+}+b_{i} w^{+} \partial_{y_{i}} w^{+}-\beta c_{i} w^{+} \partial_{y_{i}} w^{+}-\beta d\left(w^{+}\right)^{2}\right) \equiv 0 .
$$

Now assumption (iii) implies

$$
D w^{+}=0 \quad \text { on }\left\{w^{+}>0\right\} \cap \Omega .
$$


Since $w^{+} \in C^{0,1}(\bar{\Omega})$ and $w^{+} \geq 0$ in $\Omega$ with $w^{+} \equiv 0$ on $\partial \Omega$, we conclude $w^{+} \equiv 0$ in $\Omega$; that is, $u \leq v$ in $\Omega$.

Remark 2.1. Condition (2.7), with $\boldsymbol{\xi}=\left(\boldsymbol{\xi}^{\prime}, 0\right), \boldsymbol{\xi}^{\prime} \neq 0$, implies that the nonlinear operator $Q u$ is (nonuniformly) elliptic in $\Omega$ for $u_{t}$ for any $t \in[0,1]$, i.e., the $n \times n$ matrix

$$
\left[\partial_{q_{i}} A^{j}(\boldsymbol{q}, z, \mathbf{y})\right]>0 \quad \text { (positive) }
$$

for all $(\boldsymbol{q}, z, \mathbf{y}) \in \mathcal{E}\left(u_{t}, \Omega\right)$ for any $t \in[0,1]$.

Remark 2.2. Assume that $\boldsymbol{A}(\boldsymbol{q}, z, \mathbf{y})$ and $B(\boldsymbol{q}, z, \mathbf{y})$ are $C^{1}$ on $\mathbb{R}^{n} \times \mathbb{R} \times \bar{\Omega}$. Then condition (2.7) can be replaced by the uniform ellipticity condition of operator (2.2) for the functions $u_{t}, t \in[0,1]$; that is,

$$
\lambda I \leq\left[\partial_{q_{i}} A^{j}(\boldsymbol{q}, z, \mathbf{y})\right] \leq \Lambda I \quad \text { for all }(\boldsymbol{q}, z, \mathbf{y}) \in \mathcal{E}([u, v], \Omega),
$$

where $\Lambda \geq \lambda>0$ are constants and $I$ is the $n \times n$ identity matrix. For the proof of Proposition 2.1] in such a form, we use the test function $\zeta=\left(w^{+}\right)^{\frac{1}{\beta}}$ in (2.2) for $u$ and $v$, and then follow the argument of [10, Theorem 10.7(iii)]. In such a form, Proposition 2.1] is an extension of [10, Theorem 10.7(iii)] from the case $\beta=1$ to all $\beta \in(0,1]$.

Remark 2.3. The assumptions of Proposition 2.1 do not imply the boundedness of the coefficients $a_{i j}, b_{j}, c_{i}$, and $d$ in $\Omega$, defined by (2.9). These coefficients are possibly unbounded in the case of equation (1.7) with $p(\rho)=\rho^{\gamma} / \gamma$ for $\gamma>2$ if $\rho$ defined by (1.8) tends to zero at a point of $\partial \Omega$.

\section{COMPARISON PRINCIPLES \\ FOR THE SELF-SIMILAR POTENTIAL FLOW EQUATION}

Now we apply the comparison principle established in Section 2 to the self-similar potential flow equation (1.7) in $\Omega \subset \mathbb{R}^{n}$. We assume that (1.3) and (1.15) hold for $\rho>0$.

Note that equation (1.7) has form (2.2) with

$$
\operatorname{div}(\boldsymbol{A}(D \varphi, \varphi, \mathbf{y}))+B(D \varphi, \varphi, \mathbf{y})=0,
$$

where

$$
\boldsymbol{A}(\boldsymbol{q}, z, \mathbf{y}) \equiv \boldsymbol{A}(\boldsymbol{q}, z):=\rho\left(|\boldsymbol{q}|^{2}, z\right) \boldsymbol{q}, \quad B(\boldsymbol{q}, z, \mathbf{y}) \equiv B(\boldsymbol{q}, z):=n \rho\left(|\boldsymbol{q}|^{2}, z\right)
$$

with the function $\rho\left(|\boldsymbol{q}|^{2}, z\right)$ defined by (1.8), $\boldsymbol{q}=\left(q_{1}, \ldots, q_{n}\right) \in \mathbb{R}^{n}, z \in \mathbb{R}$, and $\mathbf{y} \in$ $\mathbb{R}^{n}$. We restrict to $\operatorname{such}(\boldsymbol{q}, z)$ that (1.8) is defined, i.e., satisfying $K-\left(z+\frac{1}{2}|\boldsymbol{q}|^{2}\right) \geq 0$.

Using the function $\hat{\rho}$ defined by (1.9), we can rewrite (3.2) as

$$
\boldsymbol{A}(\boldsymbol{q}, z):=\hat{\rho}\left(s_{\boldsymbol{q}, z}\right) \boldsymbol{q}, \quad B(\boldsymbol{q}, z):=n \hat{\rho}\left(s_{\boldsymbol{q}, z}\right),
$$

with

$$
s_{\boldsymbol{q}, z}:=K-\left(z+\frac{1}{2}|q|^{2}\right),
$$

and consider only those $(\boldsymbol{q}, z) \in \mathbb{R}^{n} \times \mathbb{R}$ for which $s_{\boldsymbol{q}, z}>h(0)$. Now we find by an explicit calculation that matrix (2.5), for equation (1.7) in dimension $n$ and with

$$
\beta=\frac{1}{n},
$$


is the following $(n+1) \times(n+1)$ matrix:

$$
H(\boldsymbol{q}, z)=\left[\begin{array}{ccccc}
\hat{\rho}-\hat{\rho}^{\prime} q_{1}^{2} & -\hat{\rho}^{\prime} q_{1} q_{2} & \cdots & -\hat{\rho}^{\prime} q_{1} q_{n} & \hat{\rho}^{\prime} q_{1} \\
-\hat{\rho}^{\prime} q_{1} q_{2} & \hat{\rho}-\hat{\rho}^{\prime} q_{2}^{2} & \cdots & -\hat{\rho}^{\prime} q_{2} q_{n} & \hat{\rho}^{\prime} q_{2} \\
\cdot & \cdot & \cdots & \cdot & \cdot \\
-\hat{\rho}^{\prime} q_{1} q_{n} & -\hat{\rho}^{\prime} q_{2} q_{n} & \cdots & \hat{\rho}-\hat{\rho}^{\prime} q_{n}^{2} & \hat{\rho}^{\prime} q_{n} \\
-\hat{\rho}^{\prime} q_{1} & -\hat{\rho}^{\prime} q_{2} & \cdots & -\hat{\rho}^{\prime} q_{n} & \hat{\rho}^{\prime}
\end{array}\right],
$$

where $\left(\hat{\rho}, \hat{\rho}^{\prime}\right):=\left(\hat{\rho}, \hat{\rho}^{\prime}\right)\left(s_{\boldsymbol{q}, z}\right)$.

Define $c^{2}\left(|q|^{2}, z\right):=p^{\prime}\left(\rho\left(|q|^{2}, z\right)\right)$. Then (1.11) holds. Now we write (3.5) as

$$
H(\boldsymbol{q}, z)=\hat{\rho}^{\prime}\left(s_{\boldsymbol{q}, z}\right)\left[\begin{array}{ccccc}
c^{2}-q_{1}^{2} & -q_{1} q_{2} & \cdots & -q_{1} q_{n} & q_{1} \\
-q_{1} q_{2} & c^{2}-q_{2}^{2} & \cdots & -q_{2} q_{n} & q_{2} \\
\cdot & \cdot & \cdots & \cdot & \cdot \\
-q_{1} q_{n} & -q_{2} q_{n} & \cdots & c^{2}-q_{n}^{2} & q_{n} \\
-q_{1} & -q_{2} & \cdots & -q_{n} & 1
\end{array}\right] .
$$

In particular,

$$
\sum_{i, j=1}^{n+1} H_{i j}(\boldsymbol{q}, z) \xi_{i} \xi_{j}=\hat{\rho}^{\prime}\left(s_{\boldsymbol{q}, z}\right)\left(\left(c^{2}\left|\boldsymbol{\xi}^{\prime}\right|^{2}-\left(\boldsymbol{q} \cdot \boldsymbol{\xi}^{\prime}\right)^{2}\right)+\xi_{n+1}^{2}\right)
$$

for all $\boldsymbol{\xi}=\left(\boldsymbol{\xi}^{\prime}, \xi_{n+1}\right) \in \mathbb{R}^{n+1}$ with $\boldsymbol{\xi}^{\prime}=\left(\xi_{1}, \ldots, \xi_{n}\right) \in \mathbb{R}^{n}$.

Lemma 3.1. Let $p \in C([0, \infty)) \cap C^{3}((0, \infty))$ satisfy (1.3) on $\{\rho>0\}$. Let $(\boldsymbol{q}, z) \in$ $\mathbb{R}^{n} \times \mathbb{R}$ satisfy:

(i) $\rho\left(|\boldsymbol{q}|^{2}, z\right)>0$, for $\rho\left(|\boldsymbol{q}|^{2}, z\right)$ defined by (1.8) -(1.9);

(ii) $|\boldsymbol{q}|<c\left(|\boldsymbol{q}|^{2}, z\right)$, i.e., equation (1.7) is elliptic for $(\boldsymbol{q}, z)$.

Then matrix (3.6) satisfies (2.6)-(2.7).

Proof. By (1.10) and assumption (ii), we obtain $\hat{\rho}^{\prime}\left(s_{\boldsymbol{q}, z}\right)>0$. Then, using the Schwartz inequality $\left(\boldsymbol{q} \cdot \boldsymbol{\xi}^{\prime}\right)^{2} \leq|\boldsymbol{q}|^{2}\left|\boldsymbol{\xi}^{\prime}\right|^{2}$, we find from (3.7) that

$$
\begin{aligned}
\sum_{i, j=1}^{n+1} H_{i j}(\boldsymbol{q}, z) \xi_{i} \xi_{j} & =\hat{\rho}^{\prime}\left(s_{\boldsymbol{q}, z}\right)\left(\left(c^{2}\left|\boldsymbol{\xi}^{\prime}\right|^{2}-\left(\boldsymbol{q} \cdot \boldsymbol{\xi}^{\prime}\right)^{2}\right)+\hat{\rho} \xi_{n+1}^{2}\right) \\
& \geq \hat{\rho}^{\prime}\left(s_{\boldsymbol{q}, z}\right)\left(\left(c^{2}-|\boldsymbol{q}|^{2}\right)\left|\boldsymbol{\xi}^{\prime}\right|^{2}+\xi_{n+1}^{2}\right)
\end{aligned}
$$

for all $\boldsymbol{\xi}=\left(\boldsymbol{\xi}^{\prime}, \xi_{n+1}\right) \in \mathbb{R}^{n+1}$ with $\boldsymbol{\xi}^{\prime}=\left(\xi_{1}, \ldots, \xi_{n}\right) \in \mathbb{R}^{n}$. Since $\hat{\rho}^{\prime}\left(s_{\boldsymbol{q}, z}\right)>0$, assumption (iii) implies that the last expression in (3.8) is nonnegative for all $\boldsymbol{\xi}=$ $\left(\boldsymbol{\xi}^{\prime}, \xi_{n+1}\right) \in \mathbb{R}^{n+1}$ and strictly positive if $\boldsymbol{\xi}^{\prime} \neq 0$.

Lemma 3.2. Let $p \in C([0, \infty)) \cap C^{3}((0, \infty))$ satisfy (1.3) on $\{\rho>0\}$. Then:

(i) The set $\left\{(\boldsymbol{q}, z) \in \mathbb{R}^{n} \times \mathbb{R}: s_{\boldsymbol{q}, z}>h(0)\right\}$ is convex.

(ii) The function $(\boldsymbol{q}, z) \rightarrow|\boldsymbol{q}|^{2}-c^{2}(\boldsymbol{q}, z)$ is convex on $\left\{(\boldsymbol{q}, z) \in \mathbb{R}^{n} \times \mathbb{R}: s_{\boldsymbol{q}, z}>\right.$ $h(0)\}$ if and only if (1.15) holds.

Proof. Assertion (ii) follows from (3.4).

Next, we turn to prove assertion (iii). We first calculate the second derivatives of $c^{2}\left(|q|^{2}, z\right)$. From (1.4), $c^{2}(\rho)=p^{\prime}(\rho)$. Then, from (1.4) and (1.10),

$$
\hat{\rho}^{\prime}(s)=\frac{\hat{\rho}(s)}{p^{\prime}(\hat{\rho}(s))} \quad \text { for } s>h(0) .
$$


Using (3.4), we write (1.11) as

$$
c^{2}\left(|\boldsymbol{q}|^{2}, z\right)=\hat{c}^{2}\left(s_{\boldsymbol{q}, z}\right),
$$

where $\hat{c}^{2}(s)=\frac{\hat{\rho}(s)}{\hat{\rho}^{\prime}(s)}$ for $s>h(0)$. From (3.9),

$$
\begin{aligned}
& \hat{c}^{2}(s)=p^{\prime}(\hat{\rho}(s)), \\
& \left(\hat{c}^{2}\right)^{\prime}(s)=p^{\prime \prime}(\hat{\rho}(s)) \hat{\rho}^{\prime}(s)=\frac{\hat{\rho}(s) p^{\prime \prime}(\hat{\rho}(s))}{p^{\prime}(\hat{\rho}(s))} .
\end{aligned}
$$

Then, using the notation $F(\rho)=\frac{\rho p^{\prime \prime}(\rho)}{p^{\prime}(\rho)}$, we obtain

$$
\left(\hat{c}^{2}\right)^{\prime}(s)=F(\hat{\rho}(s)), \quad\left(\hat{c}^{2}\right)^{\prime \prime}(s)=F^{\prime}(\hat{\rho}(s)) \hat{\rho}^{\prime}(s)=F^{\prime}(\hat{\rho}(s)) \frac{\hat{\rho}(s)}{p^{\prime}(\hat{\rho}(s))} .
$$

Let $(\boldsymbol{q}, z)$ satisfy $s_{\boldsymbol{q}, z}>h(0)$. Then $\rho\left(|\boldsymbol{q}|^{2}, z\right)=\hat{\rho}\left(s_{\boldsymbol{q}, z}\right)>0$. Using (3.10)-(3.11), we find that, for $i, j=1, \ldots, n$,

$$
\begin{aligned}
& \partial_{q_{i} q_{j}} c^{2}\left(|\boldsymbol{q}|^{2}, z\right)=-F\left(\hat{\rho}\left(s_{\boldsymbol{q}, z}\right)\right) \delta_{i j}+F^{\prime}\left(\hat{\rho}\left(s_{\boldsymbol{q}, z}\right)\right) \frac{\hat{\rho}\left(s_{\boldsymbol{q}, z}\right)}{p^{\prime}\left(\hat{\rho}\left(s_{\boldsymbol{q}, z}\right)\right)} q_{i} q_{j}, \\
& \partial_{q_{i} z} c^{2}\left(|\boldsymbol{q}|^{2}, z\right)=F^{\prime}(\hat{\rho}(s)) \frac{\hat{\rho}\left(s_{\boldsymbol{q}, z}\right)}{p^{\prime}\left(\hat{\rho}\left(s_{\boldsymbol{q}, z}\right)\right)} q_{i}, \\
& \partial_{z z} c^{2}\left(|\boldsymbol{q}|^{2}, z\right)=F^{\prime}\left(\hat{\rho}\left(s_{\boldsymbol{q}, z}\right)\right) \frac{\hat{\rho}\left(s_{\boldsymbol{q}, z}\right)}{p^{\prime}\left(\hat{\rho}\left(s_{\boldsymbol{q}, z}\right)\right)} .
\end{aligned}
$$

Then, using the notation $\partial_{q_{n+1}}:=\partial_{z}$, we obtain that, for any vector $\left(\boldsymbol{\xi}, \xi_{n+1}\right) \in$ $\mathbb{R}^{n+1}$ with $\boldsymbol{\xi}=\left(\xi_{1}, \ldots, \xi_{n}\right) \in \mathbb{R}^{n}$,

$$
\begin{aligned}
I & :=\sum_{i, j=1}^{n+1} \partial_{q_{i} q_{j}}\left(|\boldsymbol{q}|^{2}-c^{2}\left(|\boldsymbol{q}|^{2}, z\right)\right) \xi_{i} \xi_{j} \\
& =\left(2+F\left(\hat{\rho}\left(s_{\boldsymbol{q}, z}\right)\right)\right)|\boldsymbol{\xi}|^{2}-F^{\prime}\left(\hat{\rho}\left(s_{\boldsymbol{q}, z}\right)\right) \frac{\hat{\rho}\left(s_{\boldsymbol{q}, z}\right)}{p^{\prime}\left(\hat{\rho}\left(s_{\boldsymbol{q}, z}\right)\right)}\left(\boldsymbol{q} \cdot \boldsymbol{\xi}+\xi_{n+1}\right)^{2} .
\end{aligned}
$$

Now we prove assertion (ii). If (1.15) holds, then, using also (1.3) and (1.10), we conclude that $I$ is nonnegative. Thus, the function $|\boldsymbol{q}|^{2}-c^{2}\left(|\boldsymbol{q}|^{2}, z\right)$ is convex on $\left\{(\boldsymbol{q}, z) \in \mathbb{R}^{n} \times \mathbb{R}: s_{\boldsymbol{q}, z}>h(0)\right\}$.

Conversely, if the function $|\boldsymbol{q}|^{2}-c^{2}\left(|\boldsymbol{q}|^{2}, z\right)$ is convex on $\left\{(\boldsymbol{q}, z) \in \mathbb{R}^{n} \times \mathbb{R}\right.$ : $\left.s_{\boldsymbol{q}, z}>h(0)\right\}$, then $I \geq 0$ for any $(\boldsymbol{q}, z)$ with $s_{\boldsymbol{q}, z}>h(0)$ and $\left(\boldsymbol{\xi}, \xi_{n+1}\right) \in \mathbb{R}^{n+1}$. Let $\rho>0$. In view of (1.10) and (3.4), there exists $(\boldsymbol{q}, z)$ such that $s_{\boldsymbol{q}, z}>h(0)$ and $\hat{\rho}\left(s_{\boldsymbol{q}, z}\right)=\rho$. Fix such $(\boldsymbol{q}, z)$. Choose $\boldsymbol{\xi} \neq 0$ and $\xi_{n+1}=-\boldsymbol{q} \cdot \boldsymbol{\xi}$. Then, for such a choice of $\boldsymbol{q}, z, \boldsymbol{\xi}$, and $\xi_{n+1}$, we obtain

$$
I=\left(2+F\left(\hat{\rho}\left(s_{\boldsymbol{q}, z}\right)\right)\right)|\boldsymbol{\xi}|^{2} .
$$

Recall that $\boldsymbol{\xi} \neq 0$. Thus, $I \geq 0$ implies $F\left(\hat{\rho}\left(s_{\boldsymbol{q}, z}\right)\right)+2 \geq 0$; i.e., $F(\rho)+2 \geq 0$. Since $\rho>0$ is arbitrary, the first inequality of (1.15) holds. Also, keeping $(\boldsymbol{q}, z)$ as above and choosing $\boldsymbol{\xi}=0$ and $\xi_{n+1}=1$, we find from (3.12) that

$$
I=-F^{\prime}\left(\hat{\rho}\left(s_{\boldsymbol{q}, z}\right)\right) \frac{\hat{\rho}\left(s_{\boldsymbol{q}, z}\right)}{p^{\prime}\left(\hat{\rho}\left(s_{\boldsymbol{q}, z}\right)\right)} \text {. }
$$


Now, $I \geq 0$, 1.3) and (1.10) imply $F^{\prime}\left(\hat{\rho}\left(s_{\boldsymbol{q}, z}\right)\right) \leq 0$, i.e., $F^{\prime}(\rho) \leq 0$. Thus the second inequality in (1.15) holds for all $\rho>0$.

Now we show the weak comparison principle for the self-similar potential flow equation for polytropic gases.

Theorem 3.1 (Weak Comparison Principle). Let $p \in C([0, \infty)) \cap C^{3}((0, \infty))$ satisfy (1.3) and (1.15) on $\{\rho>0\}$. Let $\Omega \subset \mathbb{R}^{n}$ be an open bounded set. Let $u, v \in$ $C^{2}(\Omega) \cap C^{0,1}(\bar{\Omega})$ satisfy

$$
\mathcal{N}(u) \geq 0, \quad \mathcal{N}(v) \leq 0 \quad \text { in } \Omega,
$$

where $\mathcal{N}$ is the operator defined in (1.16). Furthermore, assume that

$$
\begin{aligned}
& \rho\left(|D u|^{2}, u\right)>0, \quad|D u|^{2}<c^{2}\left(|D u|^{2}, u\right), \\
& \rho\left(|D v|^{2}, v\right)>0, \quad|D v|^{2}<c^{2}\left(|D v|^{2}, v\right)
\end{aligned}
$$

in $\Omega$, where $\rho(\cdot, \cdot)$ and $c^{2}(\cdot, \cdot)$ are given by (1.8) and (1.11). Then, if $u \leq v$ on $\partial \Omega$, it follows that $u \leq v$ in $\Omega$.

Proof. Using (1.8)-(1.10), we can write the conditions

$$
\rho\left(|\boldsymbol{q}|^{2}, z\right)>0 \text { and }|\boldsymbol{q}|^{2}<c^{2}\left(|\boldsymbol{q}|^{2}, z\right)
$$

in the form

$$
s_{\boldsymbol{q}, z}>h(0) \text { and }|\boldsymbol{q}|^{2}-c^{2}\left(|\boldsymbol{q}|^{2}, z\right)<0
$$

respectively. Then Lemma 3.2 implies that the set of all $(\boldsymbol{q}, z)$ satisfying these inequalities forms a convex subset of $\mathbb{R}^{n} \times \mathbb{R}$, where we used the fact that $p(\rho)$ satisfies (1.3) and (1.15) on $\{\rho>0\}$. Thus, (3.14) implies that, for all $\mathbf{y} \in \Omega$ and $t \in[0,1]$,

$$
\rho\left(\left|D u_{t}(\mathbf{y})\right|^{2}, u_{t}(\mathbf{y})\right)>0, \quad\left|D u_{t}(\mathbf{y})\right|<c\left(\left|D u_{t}(\mathbf{y})\right|^{2}, u_{t}(\mathbf{y})\right),
$$

where $u_{t}=t u+(1-t) v$.

We also note that $\rho\left(|\boldsymbol{q}|^{2}, z\right)$ is $C^{3}$ in a neighborhood of any $(\boldsymbol{q}, z)$ satisfying $s_{\boldsymbol{q}, z}>0$. Then the regularity $u, v \in C^{2}(\Omega) \cap C^{0,1}(\bar{\Omega})$ and (3.14) imply that (3.13) holds in the weak sense of Definition 2.1. Again using the regularity of $u$ and $v$, (3.2), and (3.15), we conclude that condition (11) of Proposition 2.1] is satisfied for equation (1.16) and the functions $u$ and $v$. Furthermore, (3.15) and Lemma 3.1 imply that conditions (iii) and (iii) of Proposition 2.1 are satisfied with $\beta=\frac{1}{n}$.

Now our assertion directly follows from Proposition 2.1.

\section{Hopf-type LEMma AND A STRONG COMPARISON PRINCIPLE FOR SELF-SIMILAR POTENTIAL FLOW IN THE ELLIPTIC REGION}

We first establish a Hopf-type lemma for self-similar potential flow.

Lemma 4.1. Let $p \in C([0, \infty)) \cap C^{3}((0, \infty))$ satisfy (1.3) and (1.15) on $\{\rho>0\}$. Let $\Omega \subset \mathbb{R}^{n}$ be an open bounded set. Let $u, v \in C^{2}(\Omega) \cap C^{0,1}(\bar{\Omega})$ satisfy

$$
\mathcal{N}(u) \geq 0, \quad \mathcal{N}(v) \leq 0 \quad \text { in } \Omega,
$$


where $\mathcal{N}$ is the operator defined in (1.16). Assume that (1.17) holds. Let u, $v$, and $\mathbf{y}^{*} \in \partial \Omega$ satisfy

$$
\begin{aligned}
& u<v \text { in } \Omega, \quad u\left(\mathbf{y}^{*}\right)=v\left(\mathbf{y}^{*}\right) ; \\
& u, v \in C^{2}\left(\bar{\Omega} \cap B_{R}\left(\mathbf{y}^{*}\right)\right) \quad \text { for some } R>0 ;
\end{aligned}
$$$$
\text { strict inequalities in (1.17) hold at } \mathbf{y}^{*} \text {; }
$$

$\Omega$ has an interior ball at $\mathbf{y}^{*}$; that is, there exists $B_{r}\left(\mathbf{y}_{0}\right) \subset \Omega$ such

$$
\text { that } \mathbf{y}^{*} \in \partial B_{r}\left(\mathbf{y}_{0}\right) \text { for some } \mathbf{y}_{0} \in \Omega, r>0 \text {. }
$$

Then $\partial_{\boldsymbol{\nu}}(v-u)\left(\mathbf{y}^{*}\right)<0$, where $\boldsymbol{\nu}=\frac{\mathbf{y}^{*}-\mathbf{y}_{0}}{\left|\mathbf{y}^{*}-\mathbf{y}_{0}\right|}$.

Proof. By (4.2)-(4.3) and choosing $r$ in (4.4) smaller if necessary (and moving $\mathbf{y}_{0}$ to the point $\mathbf{y}^{*}-r \boldsymbol{\nu}$ so that (4.4) continues to hold), we can assume without loss of generality that there exists $\lambda>0$ such that

$$
\begin{array}{ll}
\rho\left(|D u|^{2}, u\right) \geq \lambda, & \lambda+|D u|^{2} \leq c^{2}\left(|D u|^{2}, u\right), \\
\rho\left(|D v|^{2}, v\right)>\lambda, & \lambda+|D v|^{2} \leq c^{2}\left(|D v|^{2}, v\right)
\end{array}
$$

in $\overline{B_{r}\left(\mathbf{y}_{0}\right)}$ and

$$
B_{r}\left(\mathbf{y}_{0}\right) \subset \Omega \cap B_{R}\left(\mathbf{y}^{*}\right) .
$$

Let

$$
w=v-u \text { in } B_{r}\left(\mathbf{y}_{0}\right) .
$$

Then $w>0$ in $B_{r}\left(\mathbf{y}_{0}\right)$ and $w\left(\mathbf{y}^{*}\right)=0$ by (4.1).

Also $w \in C^{2}\left(\overline{B_{r}\left(\mathbf{y}_{0}\right)}\right)$, and $w$ satisfies the equation

$$
\partial_{y_{j}}\left(a_{i j}(\mathbf{y}) \partial_{y_{i}} w+b_{i}(\mathbf{y}) w\right)+c_{i}(\mathbf{y}) \partial_{y_{i}} w+d(\mathbf{y}) w \geq 0,
$$

with the coefficients $a_{i j}, b_{i}, c_{j}$, and $d$ given by (2.9) with $\boldsymbol{A}$ and $B$ defined by (3.3). Furthermore, arguing as in the proof of Theorem 3.1, we conclude that the inequalities in (4.5) hold for each $u_{t}=t u+(1-t) v, t \in[0,1]$, in $B_{R}\left(\mathbf{y}_{0}\right)$. Furthermore, from (4.2) and (4.6), there exists $\Lambda$ such that $\left\|u_{t}\right\|_{C^{2}\left(\overline{B_{r}\left(\mathbf{y}_{0}\right)}\right)} \leq \Lambda$ for all $t \in[0,1]$. It follows that equation (4.7) is uniformly elliptic in $\overline{B_{r}\left(\mathbf{y}_{0}\right)}$ and its coefficients $a_{i j}, b_{i}, c_{j}$, and $d$ are in $C^{1}\left(\overline{B_{r}\left(\mathbf{y}_{0}\right)}\right)$.

Denoting

$$
\begin{aligned}
& \tilde{\boldsymbol{A}}=\left(\tilde{A}^{1}, \ldots, \tilde{A}^{n}\right) \quad \text { with } \tilde{A}^{i}(\boldsymbol{q}, z, \mathbf{y})=\sum_{j=1}^{n} a_{i j}(\mathbf{y}) q_{j}+b_{i}(\mathbf{y}) z, \\
& \tilde{B}(\boldsymbol{q}, z, \mathbf{y})=\sum_{i=1}^{n} c_{i}(\mathbf{y}) q_{i}+d(\mathbf{y}) z,
\end{aligned}
$$

we see that equation (4.7) has form (3.1) with $\tilde{A}$ and $\tilde{B}$. Denote by $\mathcal{N}(\cdot)$ the operator on the left-hand side of this equation. Using $a_{i j}, b_{i}, c_{j}, d \in C^{1}\left(\overline{B_{r}\left(\mathbf{y}_{0}\right)}\right)$, we obtain that any $\tilde{u}, \tilde{v} \in C^{2}\left(B_{r}\left(\mathbf{y}_{0}\right)\right) \cap C^{0,1}\left(\overline{B_{r}\left(\mathbf{y}_{0}\right)}\right)$ with $\mathcal{N}(\tilde{u}) \geq 0$ and $\mathcal{N}(\tilde{v}) \leq 0$ in $B_{r}\left(\mathbf{y}_{0}\right)$ satisfy these inequalities in the weak sense of Definition 2.1. Furthermore, also using (2.9) and Lemma 3.1, we conclude that equation (4.7) and the functions $\tilde{u}$ and $\tilde{v}$ satisfy conditions (ii)-(iii) of Proposition 2.1 in $B_{r}\left(\mathbf{y}_{0}\right)$, with $\beta=\frac{1}{n}$. Thus, from Proposition 2.1, equation (4.7) satisfies the weak comparison principle in the region $U$ for any open $U \subseteq B_{r}\left(\mathbf{y}_{0}\right)$. That is, if $\tilde{u}, \tilde{v} \in C^{2}(U) \cap C^{0,1}(\bar{U})$, if $\mathcal{N}(\tilde{u}) \geq 0$ and $\mathcal{N}(\tilde{v}) \leq 0$ in $U$, and if $\tilde{u} \leq \tilde{v}$ on $\partial U$, then $\tilde{u} \leq \tilde{v}$ in $U$. 
Now the $C^{1}\left(\overline{B_{r}\left(\mathbf{y}_{0}\right)}\right)$-regularity of the coefficients $a_{i j}, b_{i}, c_{j}$, and $d$ allows us to rewrite equation (4.7) in the nondivergence form:

$$
\sum_{i, j=1}^{n} \hat{a}_{i j} \partial_{y_{i} y_{j}} w+\sum_{i=1}^{n} \hat{b}_{i} \partial_{y_{i}} w+\hat{d} w=0 \quad \text { in } B_{r}\left(\mathbf{y}_{0}\right),
$$

where $\hat{a}_{i j}, \hat{b}_{i}, \hat{d} \in C\left(\overline{B_{r}\left(\mathbf{y}_{0}\right)}\right)$ and $\hat{a}_{i j}=a_{i j}$. Since this equation is uniformly elliptic in $B_{r}\left(\mathbf{y}_{0}\right)$ and satisfies the weak comparison principle on subregions of $B_{r}\left(\mathbf{y}_{0}\right)$, the standard proof of Hopf's lemma (as, e.g., in [10, Lemma 3.4]) implies that Hopf's lemma holds for solutions of this equation in $B_{r}\left(\mathbf{y}_{0}\right)$. Thus, $\partial_{\boldsymbol{\nu}} w\left(\mathbf{y}^{*}\right)<0$ for $w=v-u$.

Now we are in a place to establish the strong comparison principle for self-similar potential flow in the elliptic region, the main theorem.

Proof of Theorem 1.1. Since the weak comparison principle has been established in Theorem 3.1, it remains to prove the strong comparison principle. Furthermore, the Hopf-type lemma, Lemma 4.1. has also been established. Then we can follow the standard argument, with the only difference being that we need to check if the conditions of Lemma 4.1 are satisfied.

By Theorem 3.1 $u \leq v$ in $\Omega$. Denote $\Omega_{1}=\{u<v\} \cap \Omega$ and $\Omega_{2}=\{u=v\} \cap \Omega$. Then $\Omega=\Omega_{1} \cup \Omega_{2}$, and $\Omega_{1}$ is open.

Assume that both $\Omega_{1}$ and $\Omega_{2}$ are nonempty. Then there exists $\mathbf{y}^{*} \in \partial \Omega_{1} \cap \Omega$ which has an interior touching ball from the $\Omega_{1}$-side, and

$$
D(u-v)\left(\mathbf{y}^{*}\right)=0 \quad \text { since } \mathbf{y}^{*} \in \Omega_{2} \cap \Omega .
$$

At $\mathbf{y}^{*}$, all the conditions of Lemma 4.1 with respect to the region $\Omega_{1}$ are satisfied, where conditions (4.2) - (4.3) for $\mathbf{y}^{*}$ and $\Omega_{1}$ hold since $\mathbf{y}^{*}$ is an interior point of $\Omega$. Now an application of Lemma 4.1 leads to a contradiction with $D(u-v)\left(\mathbf{y}^{*}\right)=0$. This completes the proof of Theorem 1.1.

\section{COMPARISON PRINCIPLES}

FOR THE LINEARIZED POTENTIAL FLOW EQUATION

As a concluding remark, we now show the weak and strong maximum principles for the linearized potential flow equation in the elliptic region, in the case when the density of the background solution is bounded away from zero. Moreover, we do not need condition (1.15) for this result.

Denote by $\mathcal{N}(u)$ the operator defined in (1.16). Let, for $w \in C^{2}(\Omega) \cap C^{1}(\bar{\Omega})$,

$$
L w=\left.\frac{d}{d \tau} \mathcal{N}(u+\tau w)\right|_{\tau=0} ;
$$

i.e., let $L$ be the linearization operator of equation (1.7) on $u$. Then the equation $L w=0$ is

$$
\sum_{i, j=1}^{n} \partial_{y_{j}}\left(a_{i j}(\mathbf{y}) \partial_{y_{i}} w+b_{i}(\mathbf{y}) w\right)+c_{i}(\mathbf{y}) \partial_{y_{i}} w+d(\mathbf{y}) w=0
$$

with

$$
\begin{array}{ll}
a_{i j}(\mathbf{y})=\hat{\rho}-\hat{\rho}^{\prime} \partial_{y_{i}} u \partial_{y_{j}} u, & b_{j}(\mathbf{y})=-\hat{\rho}^{\prime} \partial_{y_{j}} u, \\
c_{i}(\mathbf{y})=-n \hat{\rho}^{\prime} \partial_{y_{i}} u, & d(\mathbf{y})=-n \hat{\rho}^{\prime}
\end{array}
$$


where $\left(\hat{\rho}, \hat{\rho}^{\prime}\right)=\left(\hat{\rho}, \hat{\rho}^{\prime}\right)\left(K-\left(\frac{1}{2}|D u(\mathbf{y})|^{2}+u(\mathbf{y})\right)\right)$.

Theorem 5.1. Let $p \in C([0, \infty)) \cap C^{3}((0, \infty))$ satisfy (1.3) on $\{\rho>0\}$. Let $\Omega \subset \mathbb{R}^{n}$ be an open set. Let $u \in C^{2}(\Omega) \cap C^{0,1}(\bar{\Omega})$ be a solution of the self-similar potential flow equation (1.7). Assume that there exists $\varepsilon_{0}>0$ such that

$$
\rho\left(|D u(\mathbf{y})|^{2}, u(\mathbf{y})\right) \geq \varepsilon_{0}, \quad|D u(\mathbf{y})|<c\left(|D u(\mathbf{y})|^{2}, u(\mathbf{y})\right)
$$

for all $\mathbf{y} \in \Omega$. Then the following comparison principle holds: If $L w \geq 0$ in $\Omega$ and $w \leq 0$ on $\partial \Omega$, then $w \leq 0$ in $\Omega$; moreover, either $w \equiv 0$ or $w<0$ in $\Omega$.

Proof. Since $\hat{\rho}(s)$ is $C^{3}$ in $\left\{s: s \geq h^{-1}\left(\frac{\varepsilon_{0}}{2}\right)\right\}$, it follows that the coefficients $a_{i j}, b_{i}, c_{j}$, and $d$ defined by (5.2) are in $C^{1}(\Omega) \cap L^{\infty}(\Omega)$. Then, repeating the argument in the proof of Lemma 4.1, we obtain that equation (5.1) with the coefficients in (5.2) satisfies the weak comparison principle in $\Omega$ and also satisfies Hopf's lemma in every $\Omega^{\prime} \Subset \Omega$. This implies the strong comparison principle in $\Omega$.

Remark 5.1. In Theorem 5.1, we do not need condition (1.15) because the coefficients of the linear equation are defined by (5.2); that is, we do not need to connect two solutions $u$ and $v$ by $u_{t}=t u+(1-t) v$ as in (2.9). The reason for requiring a positive lower bound for the density in Theorem 5.1 above is to make sure that the coefficients in (5.2) are bounded in $\Omega$; also see Remark 2.3.

\section{ACKNOWLEDGMENTS}

The work of Gui-Qiang G. Chen was supported in part by NSF grants DMS0935967, DMS-0807551, the Royal Society-Wolfson Research Merit Award (UK), and the UK EPSRC Science and Innovation award to the Oxford Centre for Nonlinear PDE (EP/E035027/1). The work of Mikhail Feldman was supported in part by the National Science Foundation under grant DMS-0800245.

\section{REFERENCES}

[1] M. Bae, G.-Q. Chen, and M. Feldman, Regularity of solutions to regular shock reflection for potential flow, Invent. Math. 175 (2009), 505-543. MR2471595(2010d:35247)

[2] G.-Q. Chen and M. Feldman, Global solutions to shock reflection by large-angle wedges, Annals of Math. (2) $\mathbf{1 7 1}$ (2010), 1067-1182. MR2630061

[3] G.-Q. Chen and M. Feldman, Shock reflection-diffraction and multidimensional conservation laws, Proc. Sympos. Appl. Math. 67, 25-51, AMS, Providence, RI, 2010. MR2605211

[4] G.-Q. Chen and M. Feldman, Potential Theory of Shock Reflection-Diffraction and von Neumann's Conjectures, monograph, preprint, 2011.

[5] G.-Q. Chen and M. Feldman, Shock reflection-diffraction and nonlinear partial differential equations of mixed type, Contemporary Mathematics, AMS, Providence, RI, 2011 (to appear).

[6] R. Courant and K. O. Friedrichs, Supersonic Flow and Shock Waves, Springer-Verlag, New York, 1962. MR0421279 (54:9284)

[7] C. M. Dafermos, Hyperbolic Conservation Laws in Continuum Physics, Springer-Verlag, Berlin, 2010. MR2574377

[8] V. Elling and T.-P. Liu, The ellipticity principle for steady and selfsimilar polytropic potential flow, J. Hyper. Diff. Eqs. 2 (2005), 909-917. MR2195986 (2006j:35193)

[9] V. Elling and T.-P. Liu, Supersonic flow onto a solid wedge, Comm. Pure Appl. Math. 61 (2008), 1347-1448. MR2436185 (2010g:76102)

[10] D. Gilbarg and N. Trudinger, Elliptic Partial Differential Equations of Second Order, 2nd Ed., Springer-Verlag, Berlin, 1983. MR737190 (86c:35035)

[11] J. Glimm and A. Majda, eds., Multidimensional Hyperbolic Problems and Computations, IMA Volumes in Mathematics and its Applications, 29, Springer-Verlag, New York, 1991. MR:1087068 (91h:00031) 
[12] J. Hadamard, Leçons sur la Propagation des Ondes et les Équations de l'Hydrodynamique, Hermann, Paris, 1903 (Reprinted by Chelsea, 1949).

[13] A. Majda and E. Thomann, Multidimensional shock fronts for second order wave equations, Comm. Partial Diff. Eqs. 12 (1987), 777-828. MR.890631 (88k:35130)

[14] C. S. Morawetz, Potential theory for regular and Mach reflection of a shock at a wedge, Comm. Pure Appl. Math. 47 (1994), 593-624. MR1278346 (95g:76030)

Mathematical Institute, University of Oxford, Oxford, OX1 3LB, United Kingdom and - Department of Mathematics, Northwestern University, Evanston, Illinois 60208

E-mail address: chengq@maths.ox.ac.uk

Department of Mathematics, University of Wisconsin, Madison, Wisconsin 53706-1388

E-mail address: feldman@math.wisc.edu 